\title{
Synthesis and Optical Properties of the Semiconductor Lead Sulfide Nanobelts
}

\author{
Xiao-hong Yang, ${ }^{1,2}$ Qing-Sheng Wu, ${ }_{+}^{1, *}$ Ya-Ping Ding, ${ }^{3}$ and Jin-ku Liu ${ }^{4}$ \\ ${ }^{1}$ Department of Chemistry, Tongii University, Shanghai, 200092, P. R. China. "E-mail: qswu@mail tongiiedu.cn \\ ${ }^{2}$ Department of Chemistry. Chizhou Teacher College, Chizhou, Anhui, 247000, P. R. China \\ ${ }^{2}$ Department of Chemistry, Shanghai University, Shanghai, $200436, P$. R. China \\ ${ }^{+}$Department of Chemistry, East China University of Science and Technolog, Shanghai 200237, P. R. China \\ Received Augztst 29, 2005
}

\begin{abstract}
The semiconductor $\mathrm{PbS}$ nanobelts (width 50-120 nm and length over $3 \mu \mathrm{m}$ ) were self-assembled in a simple reverse micelle solvent system containig the surfactant of polyoxyethylene (9) dodecy ether $\left(\mathrm{C}_{12} \mathrm{E}_{4}\right)$. The nanobelts synthesized were found to possess cube galena poly-crystal structure with high purity when analyzed by ED and X-ray diffraction. Significant "blue shift" from bulk material was observed on the PbS nanobelts using photoluminescence and UV.Vis spectroscopy. $A$ mechanism involving the possible formation of nanobelts based on surfactant template was also proposed.
\end{abstract}

Key Words : Reverse micelles, Controlled synthesis, Pb\$, Nanobelt

\section{Introduction}

Due to its unique structure and superior optoelectronic properties, nanobelts have surpassed nanowires and nanotubes and attracted a lot of interest in nanomaterials research. The controlled synthesis of no-defective, oxide nanobelts under high temperature and the solid gas phase method was first described by Pan et al. ${ }^{1}$ Since the one-dimensional nanobelts with wide band gap were expected to have vital applications in areas such as high-efficiency electricity transmission, a series of nanobelts have been fabricated, ${ }^{2}$ most of which were oxides, such as $\mathrm{ZnO}, \mathrm{SnO}_{2}, \mathrm{GaO}_{2}$, $\mathrm{MoO}_{3}$, etc. ${ }^{3.5}$ There have also been a lot of interests in making nanobelts with non-oxide materials and potentially utilizing them in areas such as electronics, biology, pigment and pharmaceuticals. The most commonly study of nonoxide semiconductors focus on sulfides, such as CdS, $\mathrm{ZnS}$, $\mathrm{Bi}_{2} \mathrm{~S}_{3}, \mathrm{Ti}_{2} \mathrm{~S}$, etc. ${ }^{6-10}$ However, synthesis of nanobelts has proven to be difficult because reactions are often performed at very high temperature and require the addition of catalysts. Therefore, it is important to explore the simple and mild methods to achieve the controlled synthesis of nanobelts, preferably under room temperature.

As typical narrow band gap semiconductors, $\mathrm{PbS}$ nanomaterials possess special optoelectronic properties, which have vital applications in optical information storage, optical communication apparatus, radioactivity detector, etc. ${ }^{11 \cdot 13} \mathrm{~A}$ number of $\mathrm{PbS}$ nanomaterials with different morphologies and structures such as nanoparticles, ${ }^{1+}$ nanorods, ${ }^{15}$ nanowires, ${ }^{16}$ nanoflakes, ${ }^{17.18}$ and nanocubes ${ }^{19}$ have been synthesized. However, so far there is no report so far on the synthesis of nanobelts by employing using the reverse micelles under a mild chemical reaction condition and at room temperature.

The reverse micelle soft-template method represents a simple and unique way to make nanomaterials with desired nano-sizes and two- or three-dimensional tropism. Compar- ed with nanomaterials obtained from physical-chemical methods, the products prepared in reverse micelles are widely considered to show superior structures and physical properties. ${ }^{20}$ Herein, this paper describes a successfully selfcontrolled synthesis of $\mathrm{PbS}$ nanobelts in a reverse micelle system by employing the surfactant of $\mathrm{C}_{12} \mathrm{E}_{9}$ as the soft template. The optical properties of $\mathrm{PbS}$ nanobelts are investigated and the potential mechanism of reaction is also proposed.

\section{Experimental Section}

Reagents and Equipment. $\mathrm{C}_{12} \mathrm{E}_{9}$ [polyoxyethylene (9) dodecy ether], cyclohexane, absolute ethanol, $n$-pentanol, $\mathrm{Na}_{2} \mathrm{~S} \cdot 9 \mathrm{H}_{2} \mathrm{O}$ and $\mathrm{Pb}\left(\mathrm{CH}_{3} \mathrm{COO}\right)_{2} \cdot 3 \mathrm{H}_{2} \mathrm{O}$ were purchased from Shanghai Chemical Reagent Factory, China. All the reagents are of analytical grade.

The morphologies of theproducts were observed through transmission electron microscopy (TEM). The crystal phase and structure of the products were detemined by X-ray powder diffraction (XRD) using Hitachi-800 diffractometer with graphite monochromatized $\mathrm{Cu}-\mathrm{Ka}$ radiation $(\lambda=$ $0.15418 \mathrm{~mm}, 50 \mathrm{kV}, 100 \mathrm{~mA}$ ). Optical properties of products were studied by Agilent 8453 UV-Visible spectrophotometer, Perking Elmer LS-55 fluorescent spectroscopy.

Preparation of the PbS Nanobelts. The reverse micelle system was prepared by adding into a $250 \mathrm{~mL}$ volumetric glass in sequence of $28 \mathrm{~mL}$ cyclohexane, $3 \mathrm{~mL} \mathrm{C} \mathrm{C}_{12} \mathrm{E}_{9}, 1 \mathrm{~mL}$ of $0.1 \mathrm{~mol} / \mathrm{L}$ aqueous $\mathrm{Pb}\left(\mathrm{CH}_{3} \mathrm{COO}\right)_{2} \cdot 3 \mathrm{H}_{2} \mathrm{O}$ and $1 \mathrm{~mL} n$ pentanol. The same experiment was also perfomed in parallel by adding into another $250 \mathrm{~mL}$ volumetric glass in sequence of $28 \mathrm{~mL}$ cyclohexane, $3 \mathrm{~mL} \mathrm{C}_{12} \mathrm{E}_{9}$ and $1 \mathrm{~mL}$ of $1.0 \mathrm{~mol} / \mathrm{L}$ aqueous $\mathrm{Na}_{2} \mathrm{~S} \cdot 9 \mathrm{H}_{2} \mathrm{O}$. Both solutions were mixed vigorously at approximately $3000 \mathrm{rmm}$ for 15 minutes, and kept at room temperature for another five minutes. After that an equal volume of two microemulsions, one containing $\mathrm{S}^{2-}$ and the other containing $\mathrm{Pb}^{2+}$, was mixed together followed 
by gentle shaking for another 5 to 10 minutes. The fural solution was kept at room temperature for 12 hours to result in the formation of nanobelts.

A volume of $3 \mathrm{~mL}$ acetone was then added to the solution to break up the micelles. Finally, the supernatant was removed from the solution described above, the bottom solution was centrifuged at $2000 \mathrm{rpm}$ for 10 minutes, washed sequentially with acetone, ethanol and water 2-3 times each. The final product should be black precipitate insoluble in water and ethanol.

The morphology of the final product was observed using TEM. One to two drops of the microemulsion solutions containing the $\mathrm{PbS}$ products were transferred onto a copper grid that was placed on filter paper followed by air-drying. The structure of the final product was studied using $\mathrm{X}$-ray diffraction, while the optical properties of the product was determined by photoluminescence (PL) and UV-Vis spectrophotometers.

\section{Results and Discussion}

Morphologies and Structures. The TEM micrograph and electron diffraction of the $\mathrm{PbS}$ nanoproducts formed are shown in Figure 1. The products have belt shape (Figure la). The single layer nanobelts were very thin and nearly transparent. There were also some other nanobelts shown in Figure la, which were likely made of a few overlapping layers. The width of the nanobelt was found to be between 50 and $120 \mathrm{~nm}$ and the length over $3 \mathrm{~km}$. The exact thickness of the nanobelts can't be easily measured. However, it was concluded that nanobelts should be approximately 3 iun thick based on: (1) As pointed in the arrow in Figure 1a, the particles were still visible even when they were undemeath the nanobelt. This has clearly demonstrated the transparent nature of the nanobelt and subsequently shown the thickness of the nanobelt was under $5 \mathrm{~nm}$. (2) As shown in Figure $1 \mathrm{~b}$, the nanobelts could be penetrated by $100 \mathrm{kv}$ electron beam. This has proven the nanobelt was less than $20 \mathrm{~nm}$ in thickness. (3) "Blue Shift" was observed on the UV-Vis absorption spectrum of the PbS nanobelts (in Figure 3). The thickness of the nanobelts was thus determined to be around
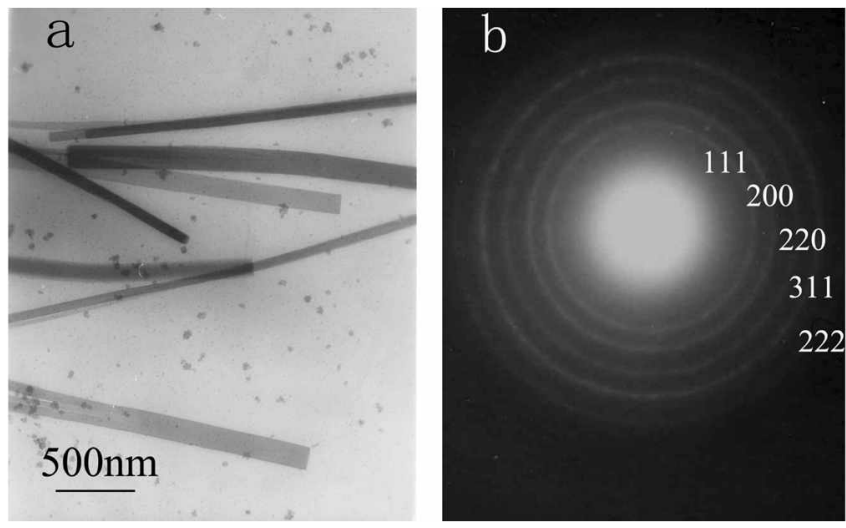

Figure 1. The TEM micrograph (a) and ED image (b) of PbS nanobelts.

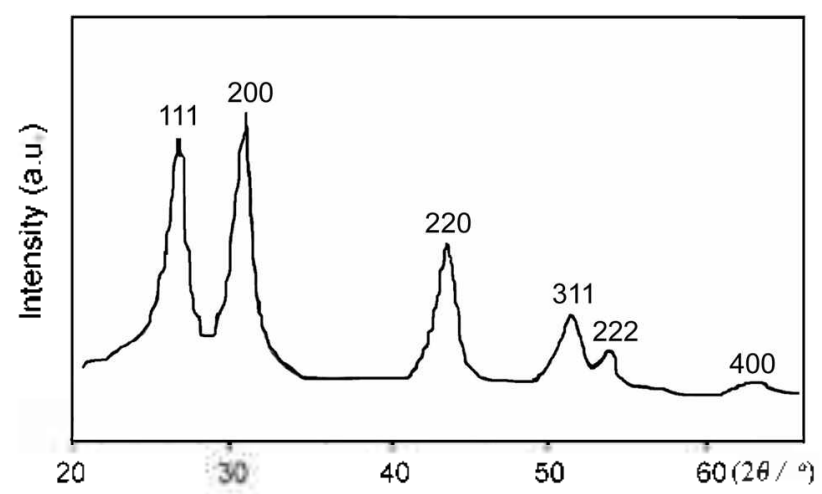

Figure 2. The XRD pattern of the $\mathrm{PbS}$ nanobelts.

$3 \mathrm{~nm}$ based on literature report. ${ }^{21}$ (4) As shown in Figure 5, the gap between different layers of micelles was nomally 1 4 nm. "- This has in turm limited the nanobelt thickness to 1-4 nin.

The comesponding electron diffraction pattem has shown the presence of clear diffraction circles, indicative of polycrystal structure. No impurity peaks were observed from the $\mathrm{XRD}$ spectral of the nanobelts (Figure 2). All the peaks found in Figure 2 matched with the ones listed in $J C$ - $P D S$ file (No: 5-592) for the cube galena structure. The lattice constant was then calculated to be $5.9378 \AA$, close to the $J C$ PDS file. Therefore, it was concluded that very pure $\mathrm{PbS}$ nanobelts were syn-thesized in this experiment and the nanobelts possessed cube galena polycrystal structure.

Experimental Conditions. Experimental conditions have been optimized in order to produce the PbS nanomaterial with the desired dimension, morphologies and structures. (1) It was found that the concentrations of reactant have influence on morphologies and structures of the products. When the concentration of $\mathrm{Pb}^{2+}$ and $\mathrm{S}^{3-}$ were $0.1 \mathrm{~mol} / \mathrm{L}$ respectively, the products were nanorods (shown in Figure 3a), not nanobelts. When the concentration of $\mathrm{Pb}^{21}$ and $\mathrm{s}^{2-}$ were $1.0 \mathrm{~mol} / \mathrm{L}$ respectively, the products were large particals (shown in Figure 3b), not nanorods or nanobelts. The excess amount of $\mathrm{S}^{2-}$ was important. It not only
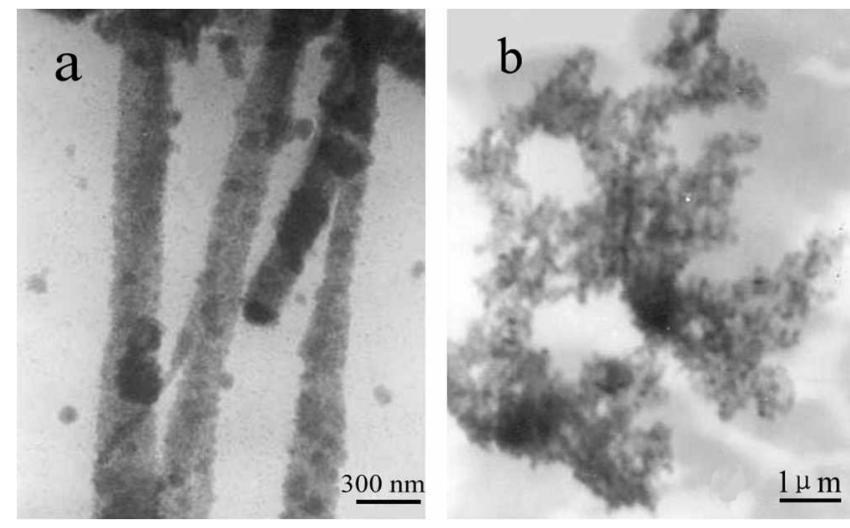

Figure 3. The TEM micrograph of PbS products (When the concentration of $\mathrm{Pb}^{2-}$ and $\mathrm{S}^{-2}$ were a $0.1 \mathrm{~mol} / \mathrm{L}$; b When the concentration of $\mathrm{Pb}^{--}$and $\mathrm{S}^{--}$were $1.0 \mathrm{~mol} / \mathrm{L}$, respectively). 
improved the morphologies of the final nanobelts, but also ensured the complete depletion of $\mathrm{Pb}^{2+}$ to avoid the environmental pollutions from heavy metal waste. In this paper the concentration ratio of $\mathrm{S}^{2-}$ to $\mathrm{Pb}^{2+}$ was $10 . \mathrm{A}$ concentration of $0.1 \mathrm{~mol} / \mathrm{L}$ for $\mathrm{Pb}\left(\mathrm{CH}_{3} \mathrm{COO}\right)_{2} \cdot 3 \mathrm{H}_{2} \mathrm{O}$ and that of $1.0 \mathrm{~mol} / \mathrm{L}$ of $\mathrm{Na}_{2} \mathrm{~S} \cdot 9 \mathrm{H}_{2} \mathrm{O}$ were used in the experiments described in this paper. (2) Molar ratio of the water to the surfactant was ten on the experiments described in this paper. Theoretically, surfactant to $n$-pentanol volume ratio $(\delta)$ had no effect on the reverse micelle system containing $\mathrm{C}_{12} \mathrm{E}_{9}$. However, a $\delta$ value of three was selected here for the solution containing $\mathrm{Pb}\left(\mathrm{CH}_{3} \mathrm{COO}\right)_{2} \cdot 3 \mathrm{H}_{2} \mathrm{O}$ to improve emulsion, and no $n$-pentanol was added to the solution containing $\mathrm{Na}_{2} \mathrm{~S} \cdot 9 \mathrm{H}_{2} \mathrm{O}$ as determined to be unuecessary.

Optical Properties. As shown in the UV-Vis absorption spectra in Figure 3, a strong absorption peak was observed at the wavelength about $347 \mathrm{rm}$ for the $\mathrm{PbS}$ nanobelts. Due to its semiconductor nature, the $\mathrm{PbS}$ nanobelts have demonstrated excellent photoluminescence (PL) properties. When excited at $390 \mathrm{~nm}$, nanobelts have shown a significant "blue shift" with the new emission wavelength peaking at $423 \mathrm{~nm}$. Unlike the bulk materials, ${ }^{23}$ the $\mathrm{PbS}$ nanobelts have shown

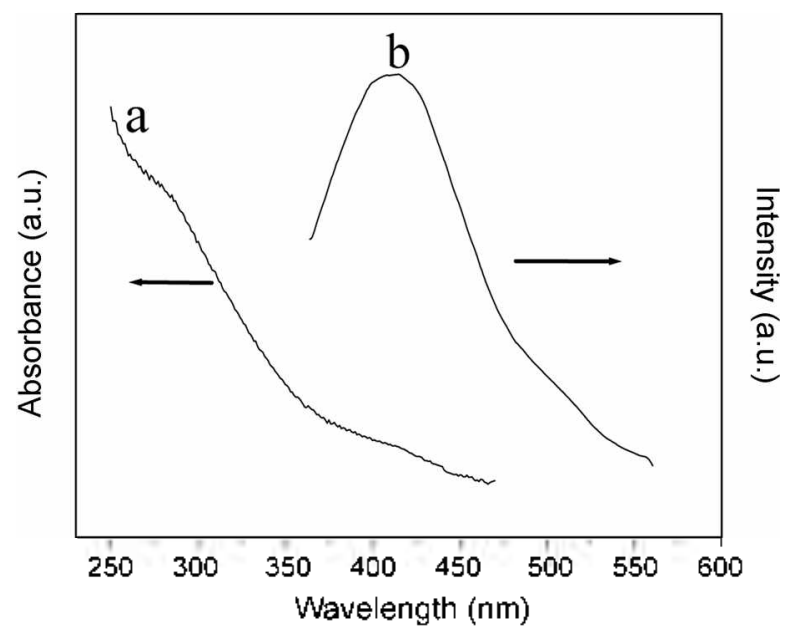

Figure 4. The LV.Vis spectra (a) and PL spectra (b) of the PbS nanobelis. narrow gap band $(0.41 \mathrm{eV})$ and large Bohr exciton radius. This has resulted in strong quantum confinement effect, unique three stage non-linear optical properties and properties of optical band restriction found on the $\mathrm{PbS}$ nanobelts.

Formation Mechanism of Nanobelts. The process of nanobelts synthesis is relatively complexed. A microemulsion template based mechanism is proposed below to explain the formation process of nanobelts in the reverse micelle system containing $\mathrm{C}_{12} \mathrm{E}_{9}$. It has been reported previously that different surfactants or even the same surfactant at different concentrations may have provided different microemulsion soft templates that resulted in the fomation of nanomaterials with different morphologies and structures, such as spheres, rods, layers, etc. ${ }^{2+}$ The surfactant of $\mathrm{C}_{12} \mathrm{E}_{9}$ is a relatively simple and symmetric molecule (Figure 4), The hydrophilic oxy-ethylene chain tended to arrange along the surface and formed the colloids of layer shape. This has subsequently provided the microemulsion template leading to the directional aggregation of $\mathrm{PbS}$ colloid nanoparticles.

$\mathrm{PbS}$ nucleuses were fomed as soon as the reverse micelles containing $\mathrm{S}^{2-}$ and $\mathrm{Pd}^{2+}$ were mixed. The nucleuses would penetrate each other and amalgamate; the $\mathrm{PbS}$ nanoparticles were then produced. Guided by the microemulsion soft template, the nanoparticles aligned along the plane surface. The gap between different layers would shrink due to the interaction between the hydrophilic groups of $\mathrm{C}_{12} \mathrm{E}_{9}$ molecules. Finally, a very thin layer of nanobelt was obtained after removing template (Figure 5).

\section{Conclusion}

The synthesis of the non-oxide nanobelts is traditionally considered to be technically very challenging. Although the reverse micelle systems have been employed to prepare nanomaterials with various morphologies and structures, the synthesis of the nanobelts using the reverse micelles isn't still reported. As described in this paper, $\mathrm{PbS}$ nanobelts were formed at the room temperature using the reverse micelles teclnnique that included the surfactant of $\mathrm{C}_{12} \mathrm{E}_{9}$. The method used is simple, easy, mild and controllable.

Significant "blue shift" was observed on the PbS nano-

$$
\mathrm{C}_{12} \mathrm{H}_{25} \mathrm{O}\left[\mathrm{CH}_{2} \mathrm{CH}_{2} \mathrm{O}\right]_{n} \mathrm{H}
$$

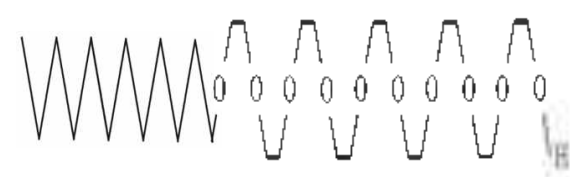

Figure 5. Chemical structure of the $C_{1:}$.
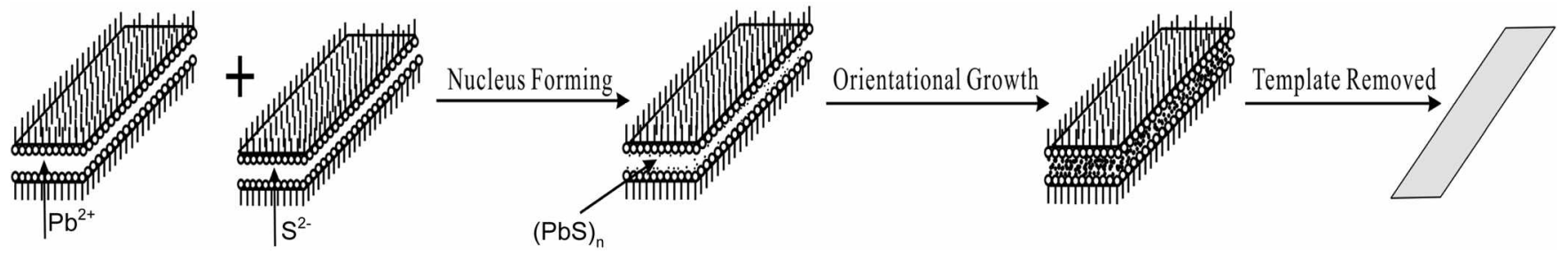

Figure 6. The proposed formation mechanism of PbS nanobelts. 
belts when analyzed by photoluminescence and UV-Vis spectroscopy. Nanobelts have thus demonstrated even more superior optoelectronic properties than nanowires and nanotubes. Therefore, they are expected to show vital applications in many important areas. A microemulsion templates based assembling process was proposed as the mechanism of synthesis. However, a lot more studies are still needed in order to fully understand the mechanism for the formation of nanobelts in the reverse micelle systems containing the surfactant of $\mathrm{C}_{12} \mathrm{E}_{9}$.

Acknowledgements. The authors are grateful to the financial support of the National Natural Science Foundation of China (Grant No: 20471042 and 20571051); NanoFoundation of Shanghai in China (Grant No: 0452nm075) and the Doctoral Program Foundation of the Ministry of Education (Grant No: 20040247045).

\section{References}

1. Pan, W. Z.; Dai, Z. R.; Wang, Z . L. Science 2001, 291, 1947.

2. Zhang, M.; Wang, Z. H.; Xi, G. C.: Ma, D.; Zhang, R.; Qian, Y. J. Crystal Gronth 2004, 268, 215.

3. Dai, Z. R.; Pan, Z. W.; Wang, Z. L. Adr. Fmo, Mater. 2003, /3, 9.

4. Chen, Y. J.; Li, Q. H.; Liang, Y, X.; Wang, T. H.; Zhao, Q.; Yu, D. P. App. Phys. Lett. 2004, $35,5682$.

5. Zhang, J.; Jiang, F.; Zhang, L. J. Phys. Chem. B 2004, $108,7002$.

6. Gao. T.: Wang. T. J. Phy. Chem. B 2004. 108. 5.

7. Liang, C. H.; Shimizu, Y.; Sasaki, T.; Umehara, H.; Koshizaki, N. J. Phy. Chem. B 2004, 108, 9728
8. Li, Y. J.: You, L. P.: Duan, R.: \$hi, P. B.: Du, H. L.: Qiao, Y. P.; Qin, G. G. Nanotechnology 2004, 15, 581.

9. Hu, P.; Liu, Y.; Fu, L.; Cao, L.; Zhu, D. J. Phs. Chent. B 2004. $108,936$.

10. Ma, B. C.: Moore, D.: Li, J.: Wang, Z. L. Ad: Mater: 2003 , 1.5 , 228.

11. Zhou, Y.: Jtoh, H.: Uemura, T.; Naka, K.: Chujo, Y. Langmin $2002,18,5287$.

12. Winiarz, J. G; Zhang, L.; Park, J.; Prasad, P. N. J. Phrs. Chent. B $2002,106,967$.

13. Leontidis, E.: Orphanou, $\mathrm{M}_{\text {.: }}$ Kyprianidou-Leodidou, T.: Krumeich, F.: Caseri, W. Nomo Lett, 2003, 3,569.

14. Zeng, Z. H.; Wang, S. H.: Yang, S. H. Cheme Mater, 1999, H, 3365.

15. Wang. S.; Yang. S. Langmuir 2000. 16. 389.

16. Ge, J. P.; Wang, A.; Zhang. H. X.; Wang, X.; Peng, Q.; Li, Y. D. Chem. Eto. J. 2005, 1/, 1889

17. Yu, D.; Wang, D.; Zhang, S.; Liu, X.; Qian, Y. J. Consfal Gronth $2003,249,195$.

18. Zhou, S. M.; Zhang, X. H.; Meng, X. M.; Fan, X.; Lee, S. T.; Wu, S. K. J. Solid Siate Chem. 2005, $778,399$.

19. Kuang, D.; Xu, A.; Fang. Y.; Liu, H.; Frommen, C.; Fenske, D. Adr: Mater: $2003,15,1747$

20. Zhang, N. W.: Wu, Q. S.; Ding, Y. P.: Li, Y. D. Chem. Lett. 2000, 6,638 .

21. Machol, J. L.; Wise, F. W.; Patel, R. C.; Tanner, D. B. Phrs. Ren: $B$ $1993,48,2819$.

22. Zhang, W. R.; Zhang. S. Y.; Zhang, Y. C. Surfactant, Antre; College Publishing Company: Hefei, 1997; p 106.

23. Daven, R. Infined, Plys. 1969, 9, 141.

24. Liu, J.; Kim, A. Y.; Wang, L. Q.; Palmer, B. J.; Chen, Y. L.; Bruinsina, P.; Bunker, B. C.; Exarhos, G. J.; Graff, G. L.; Rieke, P. C.; Fryxell, C. E.; Virden, J. W.; Tarasevich, B. J.; Chick, L. A. Act. Colloid Interface Sci. 1996, 09, 131. 